\title{
Kit didático de baixo custo utilizando Arduino para estudo e correção do efeito bouncing
}

DOI: 10.37702/2175-957X.COBENGE.2021.3731

Daniel Santos Lopes - d_santoslopes@outlook.com

Instituto Federal de Educação Ciência e Tecnologia de Minas Gerais Campus

Formiga MG

Coronel Francisco Cambraia 459

37278-000 - Santana do Jacaré - MG

Ana Flávia Peixoto de Camargos - anaflavia@ifmg.edu.br

IFMG

Rua Padre Alberico 440

35577-020 - Formiga - MG

Carlos Renato Borges dos Santos - santoscrb@gmail.com

IFTM

Rua Lourdes Rodrigues 157

35574-640 - Formiga - MG

André Roger Rodrigues - andre.roger@ifmg.edu.br

IFMG

Rua São Luiz Gonzaga, s/no , s/n

35577-010 - Formiga - MG

Paloma Maira de Oliveira - paloma.oliveira@ifmg.edu.br

IFMG

Rua Padre Alberico s/n

35577-020 - Formiga - MG

Resumo: O efeito bouncing corresponde a um fenômeno ruidoso aleatório que surge no sinal gerado por chaves mecânicas na transição de estados. Este efeito é comum em aulas laboratoriais quando se utiliza chaves e botões em projetos eletrônicos, principalmente aqueles com utilizam o Arduino, no qual muitas vezes interfere no desenvolvimento dos projetos. Muitos alunos, na maioria das vezes, não conhecem o fenômeno e, então, confunde-o com interferência, mal contato ou erro de programação. Visando demonstrar tal fenômeno e suas formas de 
mitigação, este trabalho tem como objetivo o desenvolvimento de um kit didático, de baixo custo, para auxiliar nas aulas laboratoriais e no estudo do efeito bouncing. Este estudo é desenvolvido com intuito de verificar este efeito nos sinais gerados por opto interruptores e, assim, resolver um problema de utilização do módulo interruptor óptico, denominado módulo sensor de velocidade LM393. No desenvolvimento do trabalho foi proposto uma técnica de debouncing, utilizando o kit, bem como as ferramentas Monitor Serial e Ploter Serial do Arduino IDE. Assim, vários testes foram realizados e os resultados foram satisfatórios para sanar problemas relacionados à utilização do módulo LM393.

Palavras-chave: Bouncing. Kit educacional. Arduino. LM393. 


\section{KIT DIDÁTICO DE BAIXO CUSTO UTILIZANDO ARDUINO PARA ESTUDO E CORREÇÃO DO EFEITO BOUNCING}

\section{INTRODUÇÃO}

Segundo CASTRO et al., (2009), o Ministério da Educação (MEC) "aponta que o ensino de engenharia e o conceito de formação educacional precisam desenvolver rapidamente estruturas mais flexíveis, integrando elementos de natureza multidisciplinar".

Para LIMA et al., (2008), as instituições de ensino têm enfrentado muitas dificuldades para realizar aulas laboratoriais práticas, as quais são primordiais no processo de formação do aluno. Todavia, não há recursos suficientes para disponibilizar esses materiais de ensino. Dessa forma, visando o aprendizado sobre o efeito bouncing e técnicas de debouncing foi desenvolvido um estudo para o desenvolvimento de um kit educacional que abordasse este problema em aulas laboratoriais. Em uma das aulas, este fenômeno foi identificado com uso do Arduino, em projetos eletrônicos, e a coleta do sinal não foi conforme esperada. Então, diante deste problema, foi observado que o sinal recebido poderia estar com algum ruído presente na alteração de estados e, portanto, o estudo foi aprofundado com a finalidade de detectar qual seria o motivo. Essa alteração foi caracterizada como efeito bouncing a qual está relacionado com a trepidação de chaves e foi o problema motivador desta pesquisa.

Neste sentido, o efeito bouncing pode ser definido como um fenômeno ruidoso aleatório que surge no sinal gerado por chaves mecânicas na transição de estados, tal como Figura 1. O fenômeno que ocorre na transição dos pulsos dos sinais gerados pelos opto interruptores pode ser enquadrado nessa categoria (GANSSLE, 2021) e (BRAGA, 2019). As técnicas empregadas para correção desse efeito são aperfeiçoadas ao longo do tempo de acordo com as inovações tecnológicas.

Figura 1 - Efeito Bouncing.

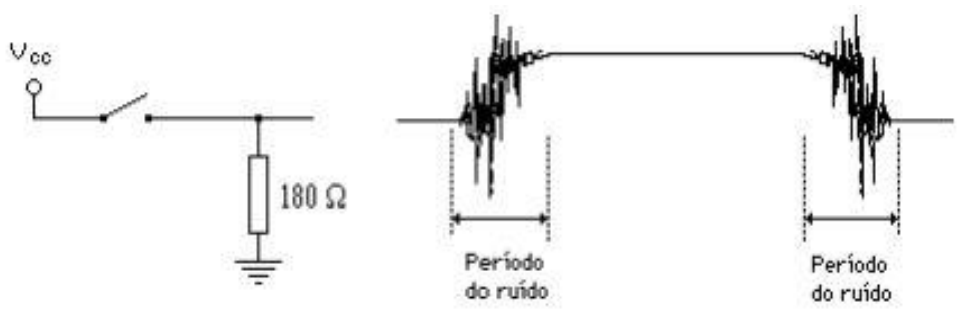

Fonte: (PINTO, 2021).

Em trabalhos acadêmicos, as técnicas de debouncing e o efeito bouncing estão presentes em estudos relacionados à eletrônica e a sistemas embarcados que utilizam chaves mecânicas. As técnicas de debouncing são empregadas de acordo com o conhecimento, a necessidade, a facilidade, a disponibilidade de componentes, dentre outros (GANSSLE, 2021), (BRAGA, 2019), (CALLAI, 2019), (MOURA, 2016) e (RAMOS, 2014).

Debouncing, por sua vez, é um termo dado às técnicas de filtragem empregadas para eliminação ou diminuição do efeito bouncing. As técnicas de correção podem ser empregadas tanto por hardware quanto por software, as quais são escolhidas em função das características do efeito e das exigências de projeto (BRAGA, 2019). Existem diversos 
tipos de circuitos empregados para correção ou filtragem do efeito bouncing por circuitos $\mathrm{RC}$, tais como: RC simples, RC com amortecedor de descarga do capacitor e RC com amortecedor de descarga do capacitor e diodo bypass. Além destes tipos, ainda existem os circuitos de debouncing schmitt trigger, que são circuitos com histerese que definem níveis de tensão para os quais deve ocorrer a comutação do sinal de saída quando o sinal de entrada aumenta ou diminui.

Idoeta e Capuano (1998) afirmam que blocos configurados com entradas schmitt trigger, "além de realizar sua função lógica, enquadra o sinal aplicado à entrada, desde que sejam respeitados os parâmetros mínimos e máximos de tensão especificados para o bloco". Dessa forma, circuitos debouncing schmitt trigger não devem funcionar bem para sinais de certos circuitos, pois os níveis do ruído atingem tensões lógicas que não são filtradas porque atendem às condições do circuito.

Portanto, este trabalho tem como contribuição e objetivo apresentar um kit didático com técnicas de debouncing para resolver problemas relacionados ao ruído em sinais gerados por chaves ópticas. Os problemas relacionados à utilização do módulo sensor LM393 foi o motivador para se aplicar técnicas de debouncing para identificar e resolver problemas no sinal gerado por opto interruptores.

\section{METODOLOGIA E MATERIAIS}

Para desenvolver o kit didático e a placa de circuitos para testes de correção do efeito bouncing, algumas etapas são importantes para a contextualização da sequência de testes realizados e serão apresentadas a seguir. Os materiais utilizados neste trabalho são os listados na Figura 2.

Figura 2 - Materiais.

\begin{tabular}{ccc}
\hline Núm. & Item & Qtde. \\
\hline 1 & Material e corte em $M D F$ & 1 \\
2 & Tacômetro digital óptico laser & 1 \\
3 & Mód. fonte alim. 3.3V 5V protob. arduino pic & 1 \\
4 & Micro motor Dc $6 V-12 V$ fase Ab codif. celo. & 1 \\
5 & Jump. macho fêmea 10cm 40 pinos para Arduino & 1 \\
6 & Foto interruptor GP1A57HRJ00F & 1 \\
7 & Mód. Chave Fim de Curso Para Ardu. & 1 \\
8 & Verniz incolor de uso geral spray & 1 \\
9 & Parafusos, porcas e arruelas & 5 \\
10 & Manípulo com porca e arruela & 1 \\
11 & Impressão a laser de esquemáticos & 10 \\
12 & Sens. Veloc. mód. encoder acopla. Óptico LM393 & 1 \\
\hline
\end{tabular}

Fonte: Próprio autor.

\subsection{Projeto da estrutura mecânica}

O desenho 2D do protótipo foi feito no software Fusion 360, da Autodesk, e a estrutura foi desenhada com o intuito de compor as seguintes partes: base principal, base para o motor de DC $12 \mathrm{~V}$ principal, base para motor DC, codificador de velocidade, disco encoder para motor DC principal e base para acomodação de sensores próximos ao disco encoder. Após realizar o desenho de cada uma das partes do projeto, foi realizado o corte das peças em material MDF, utilizando Computer numerical control (CNC) a laser. As peças cortadas podem ser visualizadas na Figura 3 e a estrutura mecânica na Figura 4. 


\section{COBENCE

Figura 3 - Peças da estrutura mecânica cortadas em MDF.

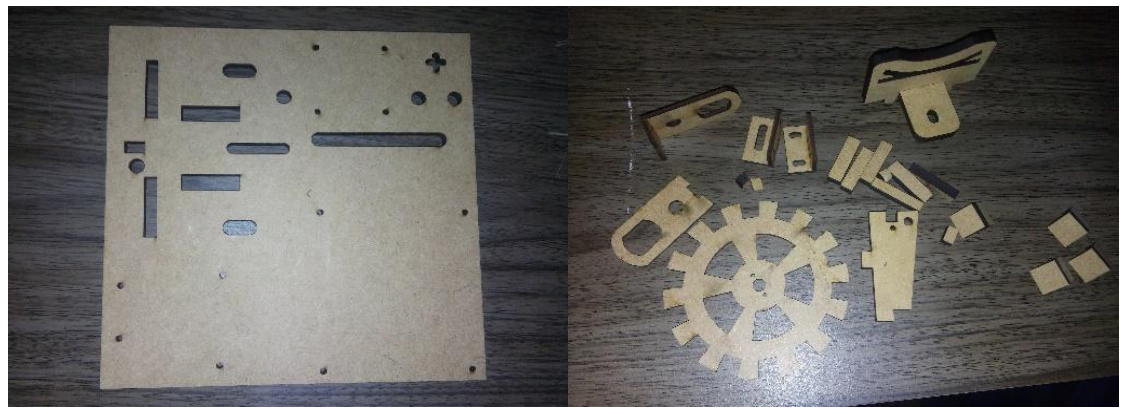

Fonte: Próprio autor.

Figura 4 - Estrutura mecânica montada.

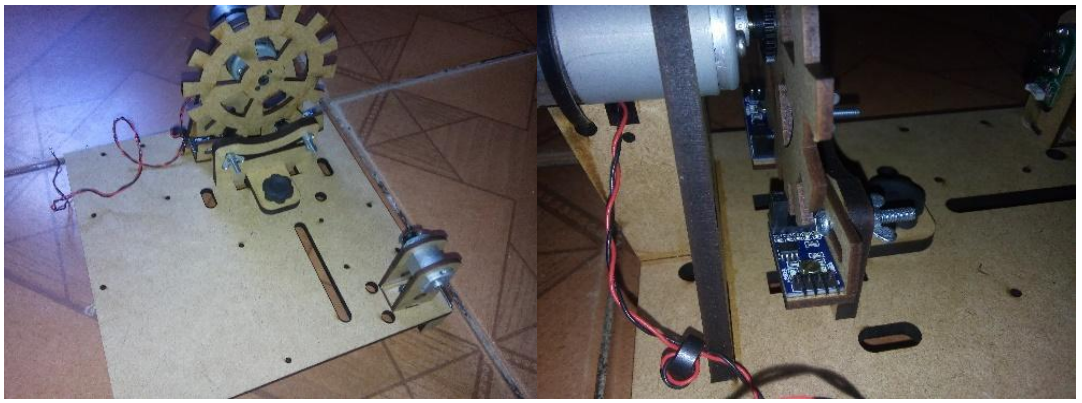

Fonte: Próprio autor.

\subsection{Circuito controlador de velocidade dos motores}

O circuito controlador de velocidade dos motores foi inserido no projeto para facilitar a realização de testes envolvendo o módulo sensor de velocidade LM393. Para isso, foi utilizada uma placa Arduino Uno com implementação lógica dedicada para recebimento dos comandos do painel e acionamento dos motores. O controle de velocidade dos motores foi feito por meio de controle PWM, utilizando o módulo ponte H L298N como atuador, o qual é capaz de controlar dois motores DC de maneira independente. Além de controlar a velocidade, este módulo permite realizar a reversão do sentido de rotação dos motores, o qual é ideal para teste na implementação do sensor de velocidade em quadratura. A Figura 5 ilustra a montagem desse circuito de controle utilizando protoboard.

Figura 5 - Circuito de comando para motores DC.

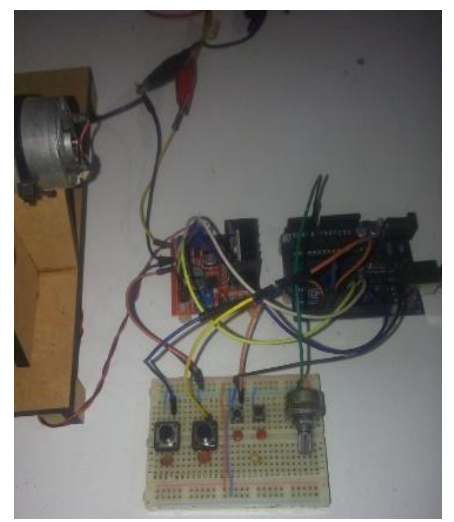

Fonte: Próprio autor. 


\subsection{Circuito didático para testes de correção do efeito bouncing}

O projeto foi desenvolvido a partir de uma placa de teste para correção do efeito bouncing por hardware utilizando resistor, capacitor e porta NAND schmitt trigger (HCF4093). Para aproveitar todas as 4 portas lógicas disponíveis no Cl HCF4093 foram desenvolvidos 4 canais de correção independentes, os quais permitem a comparação e correção de diferentes sinais. A confecção da placa foi escolhida para facilitar o manuseio e reduzir o tempo necessário para realizar os testes, não havendo necessidade do usuário se preocupar com o esquema de ligação da pinagem do $\mathrm{Cl}$, polaridade de alimentação e outros detalhes. Na Figura 6 são ilustrados os projetos da placa de circuito impresso.

Figura 6 - Projeto da placa de circuito impresso.

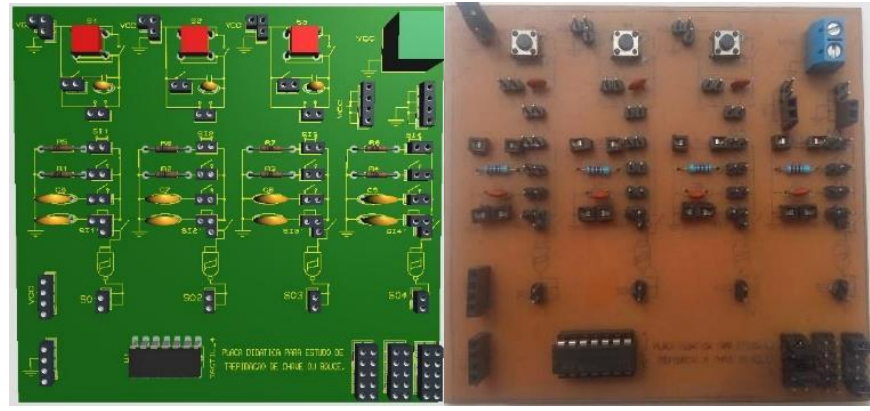

Fonte: Próprio autor

\subsection{Especificações gerais para a estrutura}

Para realizar os testes foram implementados alguns sketch's na IDE Arduino, como por exemplo: leitura de pino em flutuação, leitura de botão ou chave, contador de pulsos e medidor de velocidade simples. Após a implementação dos sketch's os testes foram realizados, começando com a leitura de pino em flutuação, com resistores de pull-up e pulldown, leitura de botões e chaves com e sem emprego de circuito de debouncing. Além disso, foram realizados testes de contador de pulsos e medidor de velocidade com sensor de velocidade LM393 e opto interruptor GP1A57HRJ00F e, por último, teste com motor codificador de velocidade.

\section{RESULTADOS}

Os testes e as análises realizadas utilizando o kit didático e a IDE Arduino são apresentados logo a seguir. Para visualizar o sinal oriundo do efeito bouncing foi utilizado a plataforma Arduino. Ao se implementar um sketch ou firmware para leitura de um botão qualquer, caso o usuário pressionar o botão uma única vez, a presença desse fenômeno é visualizada na transição de estado e faz com que o microcontrolador receba uma série de eventos de alteração de estado, pois eles operam com frequência de clock na ordem de MHz. Os testes foram coletados a partir da alteração de estado para duas chaves mecânicas, sendo uma um módulo chave fim de curso do Arduino e a outra um botão push button de dois terminais. As imagens delas podem ser visualizadas na Figura 7.

Figura 7 - Chaves mecânicas fim de curso e push button, respectivamente. 


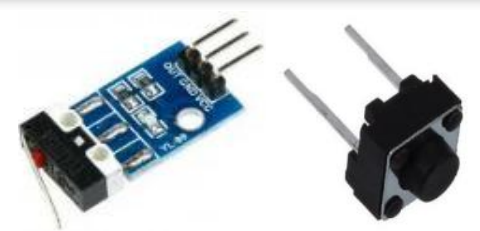

Fonte: Próprio autor.

Para a coleta dos sinais destas chaves foi necessário utilizar um protoboard. Após verificar a existência do efeito bouncing, procedeu-se com o emprego de técnicas de correção desse efeito. Foram empregadas técnicas de correção, como por exemplo: adição de capacitor de bypass ou desacoplamento, retardo por software, bem como porta NAND schmitt trigger HCF4093 presente na placa de teste projetada e módulo sensor de velocidade LM393. Neste trabalho, o foco principal consiste em apresentar a última técnica.

Assim, o primeiro teste com o módulo sensor de velocidade LM393 foi utilizando o sketch verificador de estado de entradas digitais implementado com rotina de interrupção externa do pino 2 da placa Arduino Uno. O uso desta rotina é importante porque as funções executadas por interrupção têm prioridade na execução do firmware e, portanto, tendem a reproduzir de maneira mais confiável o que ocorre com o pino. Para garantir mais confiabilidade aos testes, os acionamentos do motor DC principal e do motor codificador de velocidade foram feitos por meio do circuito de acionamento implementado que opera de forma independente, a partir de outra placa Arduino Uno. Foi necessário obter o esquema de ligação do módulo por meio de testes de continuidade com auxílio de um multímetro e da folha de dados do CI LM393. O circuito esquemático obtido é apresentado na Figura 8.

Figura 8 - Circuito esquemático do sensor de velocidade LM393.
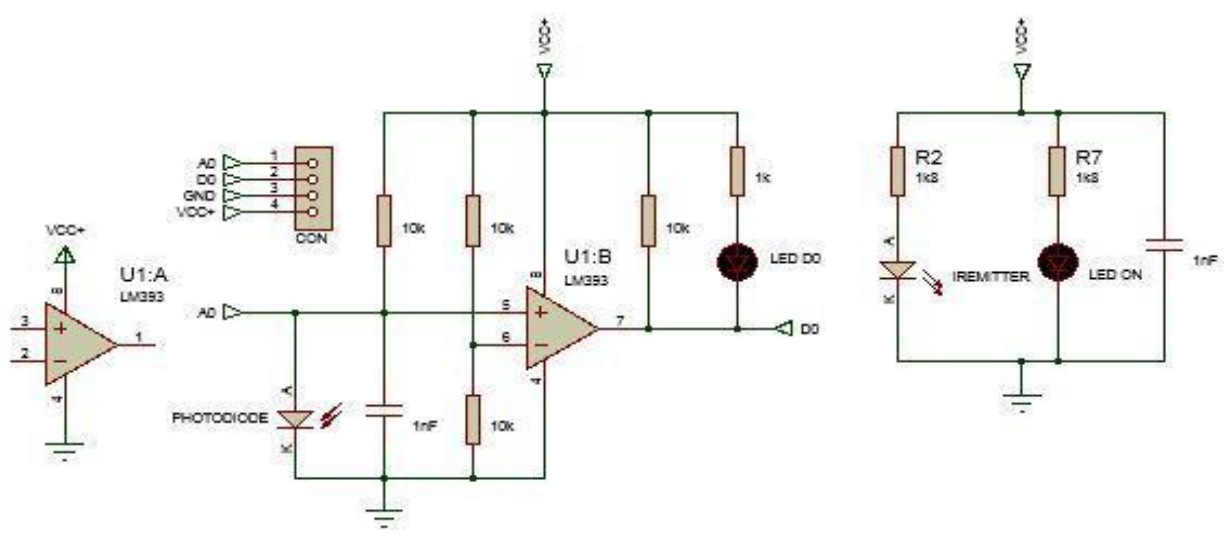

Fonte: Próprio autor.

Por meio desse esquemático, observa-se que é possível obter o sinal gerado pelo opto interruptor por meio dos pinos $\mathrm{AO}$ e DO. O pino AO corresponde ao sinal gerado pelo foto receptor, nesse caso o fotodiodo, havendo conexão com resistor pull-up de $10 \mathrm{k} \Omega$ (usado para evitar flutuação), bem como com o capacitor de desacoplamento. O pino DO corresponde ao tratamento de nível lógico do sinal AO feito com um circuito comparador, no qual é retornado nível lógico baixo para valores de $\mathrm{AO}$ menores do que $(\mathrm{VCC}+) / 2$ e nível alto para valores de $\mathrm{AO}$ maior que $(\mathrm{VCC}+) / 2$.

As saídas de sinal AO e DO foram conectadas uma por vez na placa Arduino, a qual estava conectada ao notebook, embarcada com sketch verificador de alteração de estado no pino por meio de rotina de interrupção externa. 
Optou-se por realizar testes de correção do ruído, utilizando porta lógica $N A N D$ schmitt trigger da placa de testes desenvolvida para o kit didático. As imagens das Figuras 9 e 10 ilustram as capturas dos sinais AO e DO sem correção e com correção.

Figura 9 - Sinal AO sem e com correção.

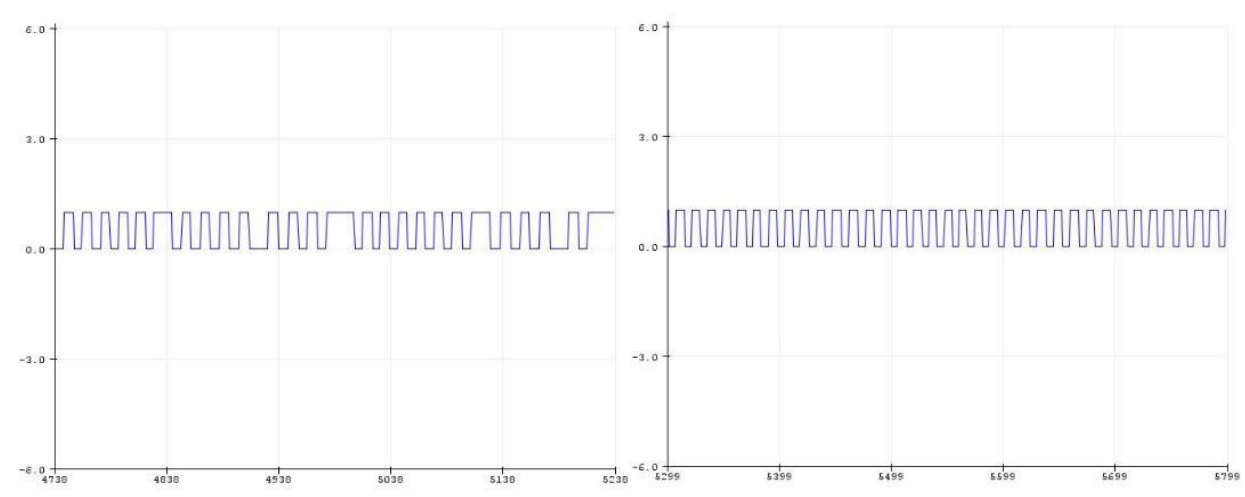

Fonte: Próprio autor.

Figura 10 - Sinal DO sem e com correção.

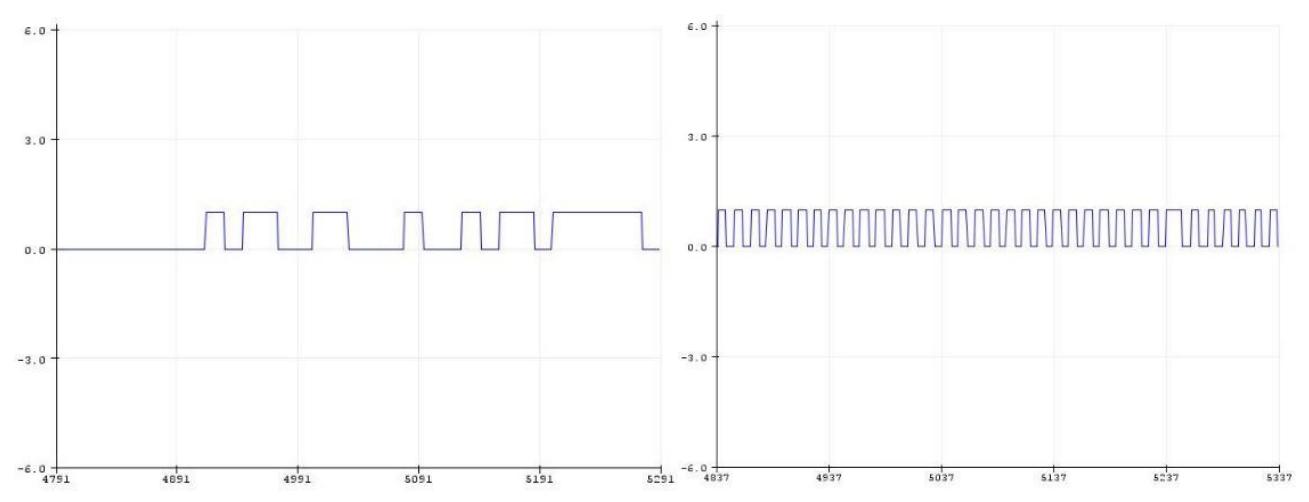

Fonte: Próprio autor.

Ao analisar as figuras acima, verifica-se que os sinais $A O$ sem e com correção schmitt trigger e o sinal DO com correção schmitt trigger apresentam formas de onda semelhantes. Já o sinal DO sem correção apresenta muitas variações na largura dos pulsos, não havendo um padrão que representa a frequência com que os pulsos são gerados. Essa variação na largura dos pulsos do sinal DO, sem correção, se deve ao fato de que o circuito comparador existente no módulo utilizado para correção de ambiguidade de nível lógico não filtra ruídos decorrentes do efeito bouncing. Assim, dependendo da intensidade de tensão, os picos deste efeito podem ser agrupados em nível alto ou baixo causando distorção do sinal. Ao ser empregada a técnica de correção schmitt trigger no sinal DO, verifica-se uma tendência de reconstituição do sinal, fazendo com que a aparência do sinal fique semelhante ao dos sinais $A O$ com e sem correção.

Nos casos em que se utilizou a porta schmitt trigger, verificou-se que os pulsos apresentavam uniformidade. Somente alguns pulsos apresentam largura ligeiramente maiores que os demais. Estes pulsos podem ser resultado da variação de velocidade do disco devido a efeitos mecânicos, como por exemplo: alinhamento do acoplamento, desbalanço devido a imperfeições no acoplamento do disco encoder e, até mesmo, variações na frequência e no ciclo de trabalho do sinal PWM gerado pela placa Arduino. 
O ciclo de trabalho do PWM para as placas Arduino varia de 0 a 255, com frequência da onda gerada de $490 \mathrm{~Hz}$ para os pinos 3, 9, 10 e 11 . Nesse projeto foi utilizado o pino 9. Entretanto, o valor mínimo do ciclo de trabalho ao qual o motor deve ser submetido depende da inércia do conjunto motor/carga. Para os testes foram utilizados ciclos de trabalho iguais a 55, 155 e 255, e para cada sinal e ciclo de trabalho foram coletados valores da rotação, em RPM, nos instantes 20s, 40s, e 60s, após a partida do motor.

Figura 11 - Sinal AO.

\begin{tabular}{cccc}
\hline & \multicolumn{3}{c}{ Velocidade(RPM) } \\
Forma medição & Duty cycle: 55 & Duty cycle: $\mathbf{1 5 5}$ & Duty cycle: 255 \\
\hline Tacômetro & 251,9 & 1169 & 1466 \\
AO Schmitt trigger & 164 & 64 & 352 \\
Tacômetro & 176 & 1165 & 1472 \\
DO & 956 & 416 & 408 \\
Tacômetro & 250,2 & 1179 & 1478 \\
DO Schmitt trigger & 540 & 744 & 72 \\
Tacômetro & 276,7 & 1189 & 1481 \\
Opto interruptor 1A57HR & 260 & 80 & 356 \\
Tacômetro & 279,4 & 1183 & 1479 \\
\hline
\end{tabular}

Fonte: Próprio autor.

Verifica-se, na Figura 11, que os valores de velocidade apresentam variação, para todos os sinais. A princípio, pequenas variações de velocidade são admissíveis, porque não há garantia de que o motor funcione de maneira uniforme com velocidade constante. Por outro lado, há discrepância entre valores de velocidade para os sinais com e sem correção schmitt trigger, tanto para $\mathrm{AO}$ quanto para DO.

A partir dos dados médios de velocidade, verifica-se a diferença entre valores de velocidade medidos por meio do sketch para os sinais AO e DO com e sem correção schmitt trigger. As diferenças entre os valores de velocidade ficam ainda maiores com o aumento do ciclo de trabalho e, consequentemente, aumento de velocidade.

Para a aferição dos sinais coletados pelo Arduino, utilizou-se um tacômetro. Conforme pode ser observado na Figura 12, os dados de velocidade medidos são muito próximos dos dados aferidos com o tacômetro.

Figura 12 - Diferença da média de velocidade da variação do duty cycle do motor após divisão por 1000ms.

\begin{tabular}{cccc}
\hline Diferança & Duty cycle: $\mathbf{5 5}$ & $\begin{array}{c}\text { Velocidade(RPM) } \\
\text { Duty cycle: } \mathbf{1 5 5}\end{array}$ & Duty cycle: $\mathbf{2 5 5}$ \\
\hline AO e tacômetro & 5,05 & 26 & 35 \\
AO Schmitt e tacômetro & 1,06 & 2,71 & 6,28 \\
DO e tacômetro & 1050,61 & 4730 & 5969,42 \\
DO Schmitt e tacômetro & 154,94 & 492,71 & 694,71 \\
1A57HR e tacômetro & 0,07 & 2,71 & 4,71 \\
\hline
\end{tabular}

Fonte: Próprio autor.

A partir dos testes e resultados foi possível verificar a eficácia do uso da correção schmitt trigger para o sensor de velocidade LM393, quando o número de pulsos por amostragem não foi fator limitante. Além deste resultado, também foi feito um outro teste para esse módulo utilizando o circuito schmitt trigger baseado em amplificador operacional. Esse circuito foi montado em protoboard utilizando o Cl LM358 com todos resistores do 
circuito iguais a $10 \mathrm{k} \Omega$. Nessa condição, os ensaios feitos mostraram que houve transição na saída quando a entrada ascendente atinge 2,8-3V e quando a entrada descendente atinge 1,8V. Esses níveis de tensão podem ser ajustados, de acordo com os valores dos resistores escolhidos. A Figura 13 ilustra a montagem desse circuito em protoboard. Os dados de velocidade para os pinos $\mathrm{AO}$ e DO do módulo com e sem correção schmitt trigger e, também, por meio do tacômetro, são apresentados na Figura 13.

Figura 13 - Diferença da média de velocidade da variação do duty cycle do motor com correção schmitt trigger utilizando o Cl LM358.

\begin{tabular}{cccc}
\hline Diferança & \multicolumn{3}{c}{ Velocidade(RPM) } \\
& Duty cycle: $\mathbf{5 5}$ & Duty cycle: $\mathbf{1 5 5}$ & Duty cycle: 255 \\
\hline AO e tacômetro & 7,42 & 13,85 & 16,71 \\
DO e tacômetro & 1100,97 & 4696,14 & 1759,71 \\
AO schm. amp. Op e tacômetro & 2,76 & 3,28 & 2,85 \\
DO schm. amp. Op e tacômetro & 0,38 & 5,28 & 1,57 \\
\hline
\end{tabular}

Fonte: Próprio autor.

Mesmo com o amplificador operacional do CI LM358 trabalhando com queda de tensão para saída de $1,5 \mathrm{~V}$, em relação a tensão máxima de alimentação, foi possível obter bons resultados com o circuito schmitt trigger implementado com esse $\mathrm{Cl}$. Verifica-se, a partir dos dados acima que o circuito foi capaz de reconstituir o sinal distorcido pelo circuito comparador presente no módulo usado como conversor de nível lógico TTL, e as diferenças desses dados em relação aos dados aferidos com o tacômetro são tão pequenas quanto as do resultado obtido para o sinal AO. Quando se compara o sinal DO (com o emprego desse circuito e sem o circuito) verifica-se uma melhoria muito significativa, pois a discrepância sem o uso de correção é muito elevada para esse sinal.

\section{CONSIDERAÇÕES FINAIS}

Neste trabalho foi apresentado o desenvolvimento de um kit educacional de baixo custo para estudar o efeito de bouncing e técnicas de debouncing. A estrutura mecânica do kit didático atendeu as expectativas de modo satisfatório. O suporte dedicado à fixação dos sensores facilitou o manuseio e a regulagem da posição deles em relação ao disco encoder. Nas situações em que o disco encoder tocava no encapsulamento do foto interruptor, devido às imperfeições no alinhamento, foi possível corrigir a posição dos módulos facilmente. $O$ fato de não ter que se preocupar com a fixação do motor e com acoplamento mecânico do disco encoder tornou a realização de testes mais simples. A utilização da plataforma Arduino permitiu a visualização dos efeitos na alteração de estados do pino de leitura provocados pelo efeito bouncing sem a necessidade de uso de aparelho osciloscópio. Também foi permitido checar a melhoria nos resultados após o emprego dos circuitos debouncing tanto para os testes com chaves mecânicas quanto nos testes realizados com os módulos que empregam dispositivo foto interruptor, comumente utilizados para medição de velocidade.

Por meio das análises e testes feitos com o módulo sensor de velocidade LM393 foi possível constatar a existência de problemas relacionados à utilização do pino de sinal DO. Foi verificado que este problema está relacionado à existência de ruídos no sinal, visto como efeito bouncing, que é potencializado pelo emprego do circuito comparador com lógica TTL, utilizado no módulo. Esse problema foi corrigido de maneira satisfatória com o emprego do circuito debouncing schmitt trigger. O sinal do pino DO pode ser totalmente corrigido através do circuito construído a partir de amplificador operacional. 
Nos testes realizados com o motor codificador de velocidade foi possível confirmar a existência dos ruídos no sinal gerado pelo opto interruptor. Os resultados obtidos mostraram que os erros provocados pela presença de ruído no sinal são muito menores que os erros vistos nos dados de velocidade obtidos por meio dos pinos AO e DO do módulo sensor de velocidade LM393. Mesmo assim, os resultados com esse módulo puderam ser melhorados com emprego do circuito debouncing schmitt trigger.

\section{REFERÊNCIAS}

ARDUINO. Volatile. [S.I.]. Disponível em: https://www.arduino.cc/reference/en/language/ variables/variable-scope-qualifiers/volatile/. Acesso em: 17 fev. 2021.

ARDUINO. ER. Serial Plotter in Arduino IDE. Disponível em: http://arduinoer.blogspot.com/2016/04/serial-plotter-in-arduino-ide.html. Acesso em: 19 fev. 2021. AUTOMALABS. A diferença entre "shield" e "módulo". Disponível em: https://www.automalabs.com.br/a-diferenca-entre-shield-e-modulo/. Acesso em: 17 de fev. 2021.

BANZI, Massimo; SHILOH, Michael. Primeiros passos com Arduino. Editora: Novatec Ltda., 2011. Disponível em: https://s3.novatec.com.br/capitulos/capitulo9788575222904.pdf. Acesso em: 08 mar. 2021.

BORGES, Marcos de Carvalho. et al. Aprendizado baseado em problemas. Medicina, SciELO Brasil. Ribeirão Preto, v. 47, n. 3, p. 301-307, 2014.

BOROCHOVICIUS, Eli; TORTELLA, Jussara Cristina Barboza. Aprendizagem baseada em problemas: um método de ensino-aprendizagem e suas práticas educativas. Ensaio: Avaliação e Políticas Públicas em Educação, 2014. Disponível em: https://www.scielo.br/pdf/ensaio/v22n83/a02v22n83.pdf. Acesso em: 18 fev. 2021.

BRAGA, Newton. C. Problemas de Repique - Como Resolver (MIC202). 2019. Disponível em: https://www.newtoncbraga.com.br/index.php/microcontroladores/143tecnologia/16303-problemas-de-repique-como-resolver-mic202.html. Acesso em: 11 fev. 2021.

CALLAI, Paulo Vinicius Gabriel. Automação de um umidificador de ar ultrassônico. 2019. TCC. Universidade Federal do Pampa. Disponível em:

http://dspace.unipampa.edu.br:8080/bitstream/riu/4825/1/Paulo\%20Vinicius\%20Gabriel\%2 0Callai\%20-\%202019.pdf. Acesso em: 17 fev. 2021.

CASTRO, Marcelo S.; MOLINARO, Luis F. R.; LIMA, Joselice F.; SANTANA, Adriano C.; RIBEIRO, Cacilda J,; CASTRO, Rosângela N, A.. Perfil do Engenheiro na Visão das Organizações de Tecnologia da Informação e de Telecomunicações de Localidades do Centro-Oeste do Brasil. Congresso Brasileiro de Educação em Engenharia, 2009, Natal. Anais.

ELETRICAL4U. Schmitt Trigger: What is it And How Does it Work? (Inverting \& NonInverting Circuit Diagrams). 2021. Disponível em: https://www.electrical4u.com/schmitttrigger/. Acesso em: 17 fev. 2021. 
GANSSLE, Jack. Guide to Debouncing - Part 2, or, How to Debounce a Contact in Two Easy Pages. 2021. Disponível em: http://www.ganssle.com/debouncing-pt2.htm. Acesso em: 17 fev. 2021.

IDOETA, Ivan V.; CAPUANO, Francisco G. Elementos de eletrônica digital. 28. ed. Editora: Érica, 1998.

LIMA, Joselice F. MARTINS, Carlos A. P. S.; TERRA Luís B. Esquema de Classificação de Laboratórios. International Conference on Information Systems and Technology Management (CONTECSI - 5). USP, São Paulo, 2008.

MORAES, Magali Aparecida Alves de; MANZINI, Eduardo José. Concepções sobre a aprendizagem baseada em proble-mas: um estudo de caso na Famema. Revista Brasileira de Educação Médica, SciELO Brasil, v. 30, n. 3, p. 125-135, 2006.

MOURA, Camila Carvalho de. Câmara térmica microcontrolada. 2017. TCC. Disponível em:

http://repositorio.roca.utfpr.edu.br/jspui/bitstream/1/10102/1/CT_COEAU_2017_1_02.pdf. Acesso em: 28 fev. 2021.

OLIVEIRA, Rodolpho Chrispim de. Conheça o Resistor de Pull Down. 2018. Disponível em: https://www.embarcados.com.br/resistor-de-pull-down/. Acesso em: 11 fev. 2021.

PINTO, Luiz Antonio Vargas. Técnicas com sistemas digitais. 2021. Disponível em: https://www.vargasp.com/download/livros/Tecnicas_digitais.pdf. Acesso em: 18 de mai. 2021.

RAMOS, Luiz F.; DALHO, Paulo Eduardo; SANTOS, Regis Eugênio. Construção de um contador de pontos de truco digital utilizando da lógica sequencial proveniente de contadores assíncronos: Relação teoria e prática. In: XLII Congresso Brasileiro de Educação em Engenharia, 2014, Juiz de Fora. Anais. Disponível em: http://www.abenge.org.br/cobenge/arquivos/5/Artigos/129126.pdf. Acesso em: 17 fev. 2021.

SILVEIRA, Cristiano Bertulucci. O que é PWM e para que serve. 2019. Disponível em: https://www.citisystems.com.br/pwm/. Acesso em: 18 fev. 2021.

SOUZA, Fábio. Entendendo as Entradas Analógicas do Arduino. 2013. Disponível em: https://www.embarcados.com.br/arduino-entradas-analogicas/. Acesso em: 17 mai. 2021.

\section{LOW COST DIDACTIC KIT USING ARDUINO FOR STUDY AND CORRECTION OF THE BOUNCING EFFECT}

Abstract: The bouncing effect corresponds to a phenomenon with random noise that appears in the signal generated by mechanical keys in the transition of states. This effect is common in laboratory classes when using keys and buttons in electronic projects, especially those using Arduino, which often interferes with the development of projects. Many students, most of the time, do not know the phenomenon and, therefore, confuse it with interference, bad contact or programming error. Aiming to demonstrate this phenomenon and its 
mitigation forms, this work aims to develop a didactic kit, at low cost, to assist in the laboratory classes and in the study of the bouncing effect. This study is developed in order to verify this effect in the signals generated by opto-switches and, thus, to solve a problem of use of the optical switch module, called LM393 speed sensor module. In the development of the work, a debouncing technique was proposed, using the kit, as well as the Arduino IDE Serial Monitor and Ploter Serial tools. Thus, several tests were carried out and the results were satisfactory to solve problems related to the use of the LM393 module.

Keywords: Bouncing. Educational kit. Arduino. LM393. 